\title{
Endoskopie zwischen Chirurgie und Gastroenterologie - aus der Sicht der Chirurgischen Arbeitsgemeinschaft für Endoskopie und Sonographie (CAES)
}

\author{
H. D. Saeger
}

Klinik und Poliklinik für Viszeral-, Thorax- und Gefäßchirurgie, Universitätsklinikum Carl Gustav Carus, Technische Universität Dresden, Deutschland

\section{Schlüsselwörter}

Chirurgische intraluminale Endoskopie - Kompetenz · Interdisziplinarität · Eigenständigkeit

\section{Zusammenfassung}

Die Endoskopie ist ein wesentlicher Bestandteil der Chirurgie. Nicht nur die Diagnostik, sondern speziell die operative Endoskopie bedarf der besonderen Kompetenz von Chirurgen. Dies wurde in der Vergangenheit in einigen Zentren eindeutig belegt. Zukünftig wird die chirurgische Endoskopie in ihrer Wertigkeit steigen. Die konventionelle Chirurgie wird schon heute zum Teil durch minimal invasive Verfahren abgelöst. Die Progredienz dieser Entwicklung ist absehbar. Dazu werden auch Kombinationen der flexiblen Endoskopie mit laparoskopischen Techniken und der Sonographie zunehmend zum Einsatz kommen. Daneben wird die Kooperation mit den Gastroenterologen intensiviert. Ein kürzlich verabschiedetes Konsensuspapier der Deutschen Gesellschaft für Verdauungsund Stoffwechselkrankheiten und der Deutschen Gesellschaft für Viszeralchirurgie unterstreicht den bilateralen Wunsch zur Zusammenarbeit. Ziele bestehen in der Optimierung der endoskopischen Leistungen. Patientenversorgung, Forschung und Lehre können so synergistisch weiter verbessert werden. Die Voraussetzung zur Durchsetzung dieser Ziele ist die gegenseitige Anerkennung der Kompetenz, die Unterstützung bei der Novellierung der Weiterbildungsordnung und die dem Bedarf und den Fortschritten angepasste Weiterentwicklung des Papiers. Daneben werden Chirurgen und Gastroenterologen die fachspezifischen Fragestellungen der intraluminalen Endoskopie auch weiterhin selbständig verfolgen.

\section{Key Words}

Surgical endoscopy · Competence · Interdisciplinary aspects · Surgical responsibility

\section{Summary}

Endoscopy between Surgery and Gastroenterology - the Point of View of the Chirurgische Arbeitsgemeinschaft für Endoskopie und Sonographie (CAES)

Flexible endoscopy is an important part of surgery. Not only diagnostic investigation, but especially operative endoscopy needs surgical competence. This has been proven in several centers. In the future the status of surgical endoscopy will increase. Already today, conventional surgery has been replaced more and more by minimal invasive procedures. This evolution probably will continue. The combination of flexible endoscopy with laparoscopy and sonography will be routinely introduced into daily surgical work. At the same time cooperation with medical gastroenterologists is intensified. A recently realized agreement of the German Society for Digestive and Metabolic Diseases and the German Society for Visceral Surgery confirms the efforts to work together in this field. The goal is to optimize endoscopic performance. Patient's care, research, and teaching can be synergistically improved. Conditions for successful consensual work are the acceptance of each others competence, the support of activities for actual education programs and the development of the agreement, depending on further necessities and progress. Besides that, specifically related research in intraluminal endoscopy will be continued by surgeons and medical gastroenterologists.

$\begin{array}{ll}\text { KARGER } & \text { (c) 2003 S. Karger GmbH, Freiburg } \\ \begin{array}{l}\text { Fax }+497614520714 \\ \text { E-mail Information@Karger.de } \\ \text { www.karger.com }\end{array} & \text { Accessible online at: } \\ \text { www.karger.com/journals/cga }\end{array}$

Prof. Dr. H. D. Saeger

Klinik und Poliklinik für Viszeral-, Thorax- und Gefäßchirurgie

Universitätsklinikum Carl Gustav Carus, Technische Universität Dresden

Fetscherstraße 74, D-01307 Dresden

Tel. +49 351 458-27 42, Fax -43 95

E-mail hans-detlev.saeger@mailbox.tu-dresden.de 


\section{Einleitung}

Die CAES vertritt die Interessen ihrer Mitglieder im Hinblick auf wissenschaftliche und berufliche Entwicklungen sowie im Bereich von Weiterbildungsfragen. Sie wurde 1976 als Arbeitsgemeinschaft der Deutschen Gesellschaft für Chirurgie für die Endoskopie gegründet, im Jahr 2000 um den Bereich Sonographie erweitert und 2002 in die Deutsche Gesellschaft für Viszeralchirurgie überführt. Die CAES gehört mit derzeit 658 Mitgliedern zu den stärksten chirurgischen Arbeitsgemeinschaften. $\mathrm{Zu}$ den Chirurgen, die in diesem Zeitraum besondere Verdienste um die Endoskopie in der Chirurgie erworben haben, gehören unter anderem die Herren Professoren H.W. Schreiber (Gründungsvorsitzender) und M. Reifferscheid, Berlin, B.C. Manegold, Mannheim, H. Troidl, Köln, und V. Schumpelick, Aachen.

Nachdem kürzlich eine eigene Arbeitsgemeinschaft für Minimal Invasive Chirurgie (CAMIC) gegründet wurde, hat die CAES die Vertretung der intrakavitären Endoskopie abgetreten und kann sich so umso intensiver den nach wie vor aktuellen Problemstellungen der endoluminalen Endoskopie widmen. Die endoluminale Endoskopie definiert sich über bestehende, natürliche Zugänge zu verschiedenen Körperhöhlen. Dies betrifft den oberen und unteren Gastrointestinaltrakt inklusive der hier einmündenden Gangsysteme, das Tracheobronchialsystem, die nach außen offenen Höhlen und Gänge des HNO-Bereichs sowie des weiblichen und männlichen Urogenitalsystems. Berührungspunkte zwischen Gastroenterologen und Chirurgen ergeben sich naturgemäß in erster Linie bei der Endoskopie des Gastrointestinaltrakts.

Nicht immer werden diese Berührungspunkte zur Bündelung von Wissen und zur Optimierung der Krankenversorgung und der gemeinsamen klinischen Forschung genutzt. Leider entsteht hier im Gegenteil nicht selten wie am Zaun zum Nachbarn oder an einigen Landesgrenzen ein «Kriegsschauplatz». Dieser ist meist auf einen Alleinvertretungsanspruch zurückzuführen, der fast nie - weder am Gartenzaun noch in der Weltpolitik - berechtigt ist.

\section{Die Grundlagen}

Bereits wiederholt wurde die historische Entwicklung der Endoskopie ausführlich dargelegt [1]. Sie wurde primär von Internisten und Chirurgen (Desormeaux, Mikulicz, Nitze, Kelling) eingeführt, bevor sich später gastroenterologische Internisten der Endoskopie zuwandten, sie zum Teil mit großem Erfolg einsetzten und neue Verfahren einführten (Demling, Classen, Ottenjann). Gleichzeitig haben Internisten und Chirurgen an der Methodenverbesserung endoskopischer Diagnostik gearbeitet und Techniken der interventionellen Endoskopie entwickelt, die konventionelle Operationen zum Teil unnötig werden ließen. Dies betrifft z. B. die Cholangiolithiasis. Der Anteil chirurgisch-operativer Gallengangsrevisionen beim Steinleiden ist heute verschwindend gering. Die endoskopische Palliation umfasst heute das Stenting von Gallenwegen, des Ösophagus und des Rektums sowie die endoluminale Tumorreduktion durch den Einsatz von Laser oder Argon-Plasma.
Darüber hinaus entstanden endoskopische Operationsverfahren, die eine endoluminale Resektion von Neoplasien des Ösophagus und des Magen-Darm-Trakts ermöglichen. Die von Bueß entwickelte transanale endoskopische Mikrochirurgie (TEM) wird heute bei richtiger Indikationsstellung zu den etablierten Verfahren gezählt. Die Mukosektomie an Ösophagus und Magen wird derzeit bezüglich ihrer Wertigkeit evaluiert (Ell, Feussner). Der kombinierte Einsatz von Sonographie und Endoskopie ist als Endosonographie zum Tumor-Staging in Ösophagus, Magen und Rektum anerkannt (Rösch, Hildebrandt). Gleiches gilt für die endoskopisch-sonographisch gestützten, internen Drainagen von Pankreaszysten und -pseudozysten. Derzeit wird der kombinierte Einsatz intraluminal endoskopischer und intrakavitär laparoskopischer bzw. thorakoskopischer Verfahren evaluiert [2]. Dafür gibt es eine Vielzahl von Ansätzen: der benigne Ösophagus- und Magenwandtumor, das lokal begrenzte Magenfrühkarzinom, gegebenenfalls zukünftig auch der breitbasige, endoskopisch allein nicht entfernbare, adenomatöse Kolonpolyp.

\section{Chirurgisch-endoskopische Spezifika}

Es gibt wohl kaum Behandlungstechniken, die nicht von Gastroenterologen, von Chirurgen und natürlich auch von anderen erlernbar wären. Voraussetzung für gute Ergebnisse sind der verantwortungsvolle Einsatz der Methode und das besondere Geschick des Therapeuten. Zusätzlich wird zur Komplikationsvermeidung zu Recht ein besonderes Gewebegefühl gefordert [3]. Dies ist nicht sui generis, wohl aber durch die Operationserfahrung im Umgang mit verschiedenen Geweben vom Chirurgen eher zu erwarten als von nichtchirurgisch tätigen Ärzten. Chirurgen sind deshalb generell zumindest als gleichwertige Endoskopiker einzustufen.

Hinzu kommen spezifische Aspekte, die den Stellenwert der chirurgischen Endoskopie unterstreichen [4]. Die klassische Situation besteht bei der Untersuchung des Rektumkarzinoms. Hier geht es neben der Diagnosesicherung um die Operationsplanung: Wird ein kontinenzerhaltendes oder lokal ablatives Verfahren durch Vollwandexzision möglich sein? Dafür ist die Einschätzung der Tumorlokalisation und -ausdehnung in longitudinaler und horizontaler Dimension erforderlich. Tumoren des «rektosigmoidalen Übergangs» müssen präoperativ dem Kolon oder Rektum zugeordnet werden, um postoperativ gegebenenfalls die adäquate adjuvante Therapie einsetzen zu können. Die Endosonographie ermöglicht die Erkennung der Infiltrationstiefe des Tumors innerhalb der Darmwand und deren Umgebung - ein wichtiges Kriterium zur Festsetzung der Reihenfolge multimodaler Therapieschemata. Die letzte Verantwortung bezüglich der Auswahl des Operationsverfahrens kann dem Operateur nicht abgenommen werden.

Die endoskopische Behandlung postoperativer Komplikationen setzt sich mehr und mehr durch. Das bedeutet nach Diagnosestellung einer Anastomoseninsuffizienz die vorsichtige, effektive Spülung der Leckage, die endoskopisch-radiologische Darstellung einer angrenzenden Insuffizienzhöhle und regelmäßige Wiederholungen der Interventionsmaßnahme. Manegold hat das Verfahren 
als «endoskopischen Verbandswechsel» bezeichnet. Bei größeren Abszessen muss ergänzend die interventionell-transkutane, eventuell sonographisch gestützte Punktion und Drainage angeschlossen werden. Wer könnte sowohl die Indikation als auch die endoskopische Behandlung besser gewährleisten als der mit der Primäroperation vertraute Chirurg?

Wenn ein nicht tastbarer, flach erhabener Tumor des Kolons lokalisiert werden muss, um die Resektionsebenen festzulegen, ist eine intraoperative Endoskopie erforderlich. Gleiches kann für eine präoperativ nicht lokalisierbare Blutung im Dünndarm oder die Überprüfung der proximalen Tumorausdehnung eines Kardiakarzinoms notwendig sein. Natürlich kann ein Internist diese Untersuchung durchführen. Zumindest aus logistischen Überlegungen ist im günstigsten Fall der Chirurg in der Lage, die Untersuchung selbst vorzunehmen.

Die Diagnostik eines zentralen Gallenwegstumors gelingt in der Regel durch endoskopische retrograde Cholangiopankreatographie mit hoher Treffsicherheit. Wirklich wertvoll ist aber die Bildgebung dann, wenn aufgrund detaillierter Darstellung des prä- und poststenotischen Gangsystems eine Operationsstrategie abgeleitet werden kann. Dies ist aber für den, der die Resektion selbst nicht durchführt, nicht von unmittelbar zwingender Bedeutung.

Schließlich wurde hin und wieder postuliert, Chirurgen seien nicht in der Lage, endoskopische Komplikationen zu beherrschen. Es fällt schwer, diesem Argument zu folgen. Wer ist eher geeignet eine Kolonperforation zu versorgen als ein Chirurg? Wer versorgt eine retroperitoneale Perforation, die anlässlich einer retrograden Gallengangsdarstellung entstanden ist? Wer kann hier besser die Indikation stellen und das Problem lösen als der mit der Endoskopie vertraute Chirurg?

\section{Interdisziplinäre Zusammenarbeit}

Die chirurgische Endoskopie ist nicht so flächendeckend verbreitet, dass die mit chirurgischen Fragestellungen verbundenen, endoskopischen, diagnostischen und therapeutischen Maßnahmen von Chirurgen allein zu lösen wären. Nach einer Umfrage von Beger und Bergmann [1] waren 2001 weniger als 5\% aller Kliniken für Allgemeine und Viszeralchirurgie (35/774) mit einer Abteilung für Endoskopie ausgestattet. Dies hat sich inzwischen zwar etwas verändert (50/774 + 12 Anträge; Stand August 2002), liegt aber nach wie vor deutlich unter $10 \%$. Die Gründe hierfür sind sicher vielschichtig, sie im Einzelnen zu analysieren wäre interessant, würde aber nicht akut zu einer Verbesserung der Situation führen.

Aus diesen Gründen ist die interdisziplinäre Zusammenarbeit von Chirurgen und Gastroenterologen erforderlich. In der täglichen Arbeit funktioniert dies je nach entgegengebrachtem gegenseitigen Verständnis sehr effektiv. Diese Form der Interdisziplinarität bedeutet aber noch keine wirkliche Zusammenarbeit in der Endoskopie. Aus der Sicht der CAES wäre es wünschenswert, in Rotationsprogrammen zwischen Gastroenterologie und Viszeralchirurgie jungen Chirurgen die Chance einer Ausbildung in der Endoskopie auch dort zu ermöglichen, wo eine chirurgische Endoskopieabteilung nicht existiert.

Auch bei bestem Willen aller Beteiligten wird diese Vorstellung nicht ohne weiteres zu verwirklichen sein. Da ist zum einen der Zwang finanzieller Ressourcen. Andererseits ist die Personalausstattung in Krankenhäusern so knapp bemessen, dass die zur Verfügung stehenden Ärzte kaum in der Lage sind, sich an Weiterbildungsprogrammen außerhalb der notwendigsten abteilungs- bzw. klinikspezifischen Aufgaben zu beteiligen. Nichtuniversitäre Klinikbetreiber sind ohnehin immer weniger bereit, die Weiterbildung junger Ärzte überhaupt zu fördern. Es wird also nicht zu einer von manchen Gastroenterologen befürchteten Übernahme der Endoskopie durch Chirurgen kommen.

Der Schwund chirurgischer Präsenz in der Endoskopie ist zu einem nicht unwesentlichen Anteil hausgemacht [5]. Vergleichbar mit der Entwicklung in der Intensivmedizin oder der Mammakarzinom-Chirurgie, haben wir uns selbst nicht ausreichend für diese Bereiche eingesetzt. So ist in den letzten Dekaden ein Kompetenzverlust aufgetreten, der die generelle Forderung auf chirurgische Zuständigkeit in diesen Gebieten nicht rechtfertigt. Andererseits gibt es äußerst aktive endoskopische Arbeitsgruppen in der Chirurgie, die nicht nur in der unmittelbaren Patientenversorgung, sondern auch in Forschung und Ausbildung bemerkenswert erfolgreich sind. Hier besteht höchste endoskopische Kompetenz, die Leistungsdaten sind exzellent, und die Gruppen verstehen es, junge Assistenten für die chirurgischen Endoskopie zu begeistern. Es ist erfreulich, anlässlich von Symposien und Workshops die Präsentationen aus diesen chirurgischen Endoskopieabteilungen zu erleben. Sie verdienen unsere Anerkennung und Förderung. Es wäre absolut kontraproduktiv, sie aus Rationalisierungsgründen mit anderen Abteilungen einer Klinik zusammenzuführen und sie dann der Leitung eines möglicherweise weniger kompetenten Gastroenterologen zu unterstellen. Durch Demotivierung der Mitarbeiter und zwangsläufig zu erwartende Reibungsverluste wird die Patientenversorgung beeinträchtigt. Insgesamt lassen sich die durch $\mathrm{Zu}$ sammenlegung verbesserten wirtschaftlichen Aspekte im Endeffekt nicht umsetzen. Die Folge kann insgesamt eine defizitäre Entwicklung sein.

Die Schaffung interdisziplinärer Endoskopieeinheiten kann durchaus sinnvoll sein. Wenn der angestrebte Effekt der Verbesserung von Arbeitsabläufen und der Leistungsqualität erreicht werden soll, muss eine den örtlichen Gegebenheiten angepasste Analyse vorangestellt und die Leitung dem kompetentesten Endoskopiker unterstellt werden. Das wird heute unter den gegebenen Umständen überwiegend ein Gastroenterologe sein, bedeutet aber in einigen Zentren, die Leitung einem Chirurgen zu übertragen. Erfahrungsgemäß ist Kompetenz in den meisten Fällen auch mit der gebotenen Fairness gegenüber dem komplementären Fachvertreter verbunden.

In diesen Abteilungen ist ein Nutzungsplan für die beteiligten Kliniken nach Stunden oder Wochentagen ebenso wie ein Dienstplan zu erstellen, der die Bereitschaftsdienste organisiert, um die Patientenversorgung über $24 \mathrm{~h}$ täglich sicherzustellen. Die Leiter vertreten sich gegenseitig, ein turnusmäßiger Wechsel der Leitung ist 
dann anzustreben, wenn die Partner gleichermaßen kompetent sind.

\section{Konsensuspapier}

Um alle diese Punkte zu fixieren, wird seit 3 Jahren an einem Konsensuspapier zwischen Gastroenterologen und Viszeralchirurgen gearbeitet. An mehreren Versionen wurde immer wieder gefeilt, der letzte Entwurf wurde kürzlich von Vertretern der Deutschen Gesellschaft für Verdauungs- und Stoffwechselkrankheiten (DGVS) und der Deutschen Gesellschaft für Viszeralchirurgie (DGVC) verabschiedet. Die Begründung für ein Konsensuspapier wurde aus der Notwendigkeit der gemeinsamen Strategie im Hinblick auf die Novellierung der Weiterbildungsordnung, die Schaffung von Synergien für Diagnostik und Therapie gemeinsamer Patienten sowie die Einrichtung interdisziplinärer Endoskopieabteilungen und nicht zuletzt kooperativer Forschungsaktivitäten abgeleitet.

Derartige Vereinbarungen sind immer durch Kompromisse geprägt. Dies führt zwangsläufig zur Unzufriedenheit von Mitgliedern beider Gesellschaften. Die wesentlichen Inhalte des Papiers betreffen die Kooperation in der Diagnostik und Behandlung von Patienten - in erster Linie bezüglich endoskopischer Maßnahmen --, die Weiterbildung sowie die Sicherung von Leistungsqualität und die Ausarbeitung von Leitlinien und Standards. Viele Kollegen haben die letzte Form als Kompromiss akzeptiert. Kritiker der Ausarbeitung sehen verschiedene Gefahren in bestimmten Inhalten und Formulierungen. Dazu gehören besonders die Zugeständnisse der Chirurgie bezüglich der Laparoskopie und der Proktologie. Die Einbeziehung bildgebender Verfahren und onkologischer Behandlungskonzepte in das Konsensuspapier wird als unnötige Eröffnung neuer Problemkreise mit anderen Fachgebieten angesehen.

Tatsächlich sind die Sorgen nicht unbegründet; erste kritische Stellungnahmen aus den benachbarten Fachgebieten liegen bereits vor. Der Wert des Papiers wird erstmals anlässlich der Verhandlungen zur Novellierung der Weiterbildungsordnung beim Ärztetag deutlich werden. Wird es eine gegenseitige Unterstützung der Anträge durch Internisten und Chirurgen geben? Bei Fokussierung auf sachliche Argumente unter Berücksichtigung der zentralen Frage «Was nützt den Patienten?» dürfte ein zufrieden stellendes Ergebnis leicht zu erreichen sein. Dieses Ziel war auch einer der hauptsächlichen Motoren zur Erarbeitung des Konsensuspapiers. Es bleibt zu hoffen, dass die Umsetzung der gesteckten Ziele Realität wird und nicht die befürchtete Durchsetzung von Eigeninteressen einzelner Fachvertreter im Vordergrund steht. Die Weiterbildungsordnung wird im vorliegenden Papier wiederholt als Rahmenbedingung zur Umsetzung verschiedener Punkte angeführt. Hier ist also höchste Aufmerksamkeit und Engagement von beiden Seiten gefordert.

Positiv gesehen, bietet die Erarbeitung gemeinsamer, konsensueller Vorstellungen erstmals die Chance, die intraluminale Endoskopie von zwei Seiten synergistisch zu fördern [6]. Alle an diesem
Thema interessierten Kollegen, Gastroenterologen und Chirurgen sind aufgefordert, an kommenden Entwicklungen der Endoskopie mitzuarbeiten, die Kompetenzen gegenseitig anzuerkennen und die mit hoher Wahrscheinlichkeit notwendigen Anpassungen des Papiers aktiv zu begleiten.

\section{Die Realität}

Die Strukturen zur Schaffung bzw. zur Organisation von Endoskopieabteilungen werden in der Regel durch die Klinik/Krankenhausträger festgelegt. Hier werden Entscheidungen von verschiedenen Voraussetzungen beeinflusst. Die Berücksichtigung personeller und finanzieller Ressourcen zwingt in den meisten Fällen zur Bildung eines Endoskopiezentrums. Die derzeitigen Modelle sehen wie folgt aus:

1) Eine endoskopische Abteilung wird internistisch oder chirurgisch betrieben.

2) In letzter Zeit wurden interdisziplinäre Zentren gebildet, in denen Internisten und Chirurgen gemeinsam arbeiten.

3) Die Klinik stellt Räume und apparative Ausstattung zur endoskopischen Nutzung für verschiedene Fachgebiete zur Verfügung. Nach einem Zeitplan wird die Nutzung für die beteiligten Endoskopiker festgelegt.

4) In großen Einrichtungen, z. B. Universitätskliniken, besteht je ein endoskopisches Zentrum für die chirurgische und die gastroenterologische Klinik.

Alle Modelle haben ihre Berechtigung und sollten nach den Gegebenheiten am Ort ausgewählt werden. Wie bereits ausgeführt, ist die Zuordnung der Abteilung nach der endoskopischen Kompetenz zu entscheiden. Wenn auch derzeit über $90 \%$ der endoskopischen Abteilungen in deutschen Kliniken internistisch-gastroenterologisch geleitet werden, kann daraus kein Automatismus abgeleitet werden.

Die Endoskopie ist nicht nur heute, sondern auch mittel- und langfristig ein existenzieller Bestandteil der Chirurgie. Seit der flächendeckenden Einführung und den Erfolgen der minimal invasiven Chirurgie ist auch die intraluminale Endoskopie zunehmend in den Mittelpunkt des Interesses operativer Therapie gerückt. Neben der intrakavitären muss auch die intraluminale Chirurgie weiterentwickelt und bezüglich ihrer Effizienz geprüft werden. Die konventionelle, offene Chirurgie wird dabei mit hoher Wahrscheinlichkeit zunehmend von Verfahren mit minimalen Zugangswegen abgelöst werden. Die Ziele und Notwendigkeiten der operativen Methoden müssen sich dabei an den evaluierten, offenen Verfahren orientieren. Deshalb muss sich die Chirurgie mehr als bisher mit den Möglichkeiten der Endoskopie - auch in Kombination mit intrakavitären (z. B. laparoskopischen) und sonographischen Verfahren - auseinandersetzen. Die Klinikleitungen sind gut beraten, diese Innovationen zu fördern und auf eine solide Basis zu stellen. In einigen Zentren wurden diese Entwicklungen bereits erkannt und durch die Schaffung chirurgisch-endoskopischer Abteilungen inklusive Forschungs- und Ausbildungsabteilungen unterstützt. 


\section{Fazit}

Die CAES vertritt die Belange und die Notwendigkeiten der Endoskopie und der Sonographie in der Chirurgie. Dazu gehört neben der Krankenversorgung die Förderung der Forschung und der Ausbildung. Derzeit sind in der Liste «Chirurgisch-endoskopische Kompetenzzentren» der DGVC 59 Abteilungen erfasst. Der Grad der Kompetenz wird nach dem Muster der chirurgischen Sonographie in einem Stufenplan differenziert werden, so dass Abteilungen, Kompetenz- und Ausbildungszentren sowie Referenzzentren unterschieden werden. Damit wird das Leistungsspektrum chirurgischer Endoskopie in Deutschland definiert werden können eine Voraussetzung, um den Anforderungen an die Leistungsqualität und zukünftigen Entwicklungen Rechnung zu tragen.

Einige Chirurgen haben sich bereits bisher mit hohem Engagement um die Entwicklung der Endoskopie und auch der Sonographie in unserem Fachgebiet besondere Verdienste erworben. Sie verdienen in hohem Maße unsere Anerkennung, Unterstützung und weitere Förderung. Jauch [7] hat unterstrichen, wie gerade in
Zeiten wirtschaftlicher Zwänge und der Prozessoptimierung die unmittelbare Verfügbarkeit der Endoskopie im perioperativen Management erforderlich ist.

Daneben sollte die Kooperation mit der Gastroenterologie intensiviert werden. Um dabei gegenseitige Akzeptanz zu erreichen, wurde ein Konsensuspapier erarbeitet, das die wesentlichen Punkte der Zusammenarbeit definiert. Es liegt in der Natur dieser Vereinbarungen, dass dabei Kompromisse von allen Beteiligten unumgänglich sind. Voraussetzung für die erfolgreiche Umsetzung von konsensuellen Bestrebungen ist die Anerkennung der Arbeit des Kooperationspartners. Die CAES, DGVC und DGVS müssen die Inhalte des Papiers überwachen und gewährleisten. Ohne Zweifel ist die Chirurgie ein kompetenter Partner, der auf die Endoskopie ebenso wenig verzichten kann wie die Gastroenterologie. In einem Kooperationskonzept sind nur gleichwertige Partner in der Lage, die Sache - hier die Endoskopie - synergistisch im Sinne der weiteren Optimierung der Patientenversorgung, der Forschung und der Lehre voranzutreiben.

\section{Literatur}

1 Beger HG, Bergmann U: Surgical endoscopy an old and new challenge for surgeons. Langenbecks Arch Surg 2001;386:161-162.

2 Siewert JR: Operation, Intervention, Rendezvousmanöver. Chirurg 2000;71:1191-1192.

3 Grund KE, Schumpelick V: Durch Kompetenz zur chirurgischen Endoskopie. Chirurg 2002;73 32-37.
4 Manegold BC: Endoskopische Chirurgie. Zehn gute Gründe zur Integration der flexiblen Endoskopie in die Chirurgie. Min Invas Chir 1997;6:7576.

5 Schumpelick V: Chirurgische Endoskopie : Wie gewonnen so zerronnen? Chirurg 2000;39:326327.

6 Riemann JF: Gastroenterologie. Neue Denkge- bäude, faszinierende Wege und Schnittstelle zur Chirurgie. Dtsch Med Wochenschr 1999;124: 1546-1549.

7 Jauch K: Chirurgische Endoskopie und Sonographie sind verstärkt zu fördern. Viszeralchirurgie 2002;37:183. 